\title{
Operating Room Door Opening Patterns in Total Hip and Knee Arthroplasties
}

\author{
Luke Reardon $^{1}$ and Tricia Blalock ${ }^{1}$
}

${ }^{1}$ Baylor University, Waco, TX, USA

DOI: https://doi.org/10.47611/jsr.v10i1.1179

\begin{abstract}
$\underline{\text { ABSTRACT }}$
Background Operating room (OR) foot traffic is a contributing factor in the development of periprosthetic joint infection (PJI), following total joint arthroplasty (TJA). Operating room door openings have been shown to increase bacterial counts in the operating room (OR), contributing to the risk of periprosthetic joint infections (PJIs). Because periprosthetic joint infections (PJIs) are a costly and challenging complication, understanding door opening behavior is essential to minimize potential of periprosthetic joint infections (PJIs). Methods An observational study was conducted to evaluate operating room (OR) foot traffic patterns for total knee and hip arthroplasties in a non-academic facility. Sixteen surgeries by 6 different surgeons were observed, 10 inpatient total hip arthroplasties (THAs) and 6 inpatient total knee arthroplasties (TKAs). Operating room (OR) traffic was monitored and recorded by counting the number of door openings, noting the timing of door openings, detailing personnel entering/exiting, and documenting the door used and the duration of each procedure. Results The average number of door openings for all cases during the possible contamination period was 73.4, and the average case length was 147 minutes. Sixty-four percent of traffic occurred from the time sterile trays were open to the incision being made and $36 \%$ of traffic occurred from incision to wound closure. Conclusion Managing door openings in the operating room (OR) is a modifiable risk factor associated with periprosthetic joint infection (PJI). From incision to closure, nurses and medical supply representatives were the largest contributors to operating room (OR) foot traffic during total joint arthroplasty (TJA) procedures. Understanding door openings during total joint arthroplasties (TJAs) will allow for the implementation of effective strategies to reduce operating room (OR) foot traffic.
\end{abstract}

\section{Introduction}

Periprosthetic joint infection (PJI) is recognized as one of the most challenging and disastrous complications associated with total joint arthroplasty (TJA), costing millions of dollars annually. Management of periprosthetic joint infections (PJIs) often requires hospital re-admissions, multiple revision surgeries, and extended rehabilitation services. Using the nation-wide in-patient sample (NIS) data from 2001-2009, Kurtz et al., found the relative incidence of periprosthetic joint infection (PJI) ranged between $2.0 \%$ and $2.4 \%$ of Total Hip Arthroplasties (THAs) and Total Knee Arthroplasties (TKAs), increasing over time. Other studies that used a narrower definition of PJI reported lower rates ranging from $0.6 \%$ to $0.9 \%$.

The demand for total joint arthroplasties (TJAs) is anticipated to increase due to the aging U.S. population and projected increases in obesity prevalence. Wolford et al., project a $14 \%$ increase in complex surgical site infections (SSIs) following hip and knee arthroplasty between 2020 and 2030 if current rates are not reduced. The economic consequences associated with treating periprosthetic infections are substantial. The annual cost of treating PJI cases in US hospitals increased from $\$ 320$ million in 2001 to $\$ 566$ million in 2009. Based on the hospital cost estimated from the 2001-2009 nation-wide in-patient sample (NIS) data, periprosthetic joint infection (PJI) cases are expected to incur a cost to US hospitals around \$1.62 billion (CI, \$1.53 - 1.72 billion) in 2020. 
The etiology of periprosthetic joint infections (PJIs) is multifaceted and incudes patient and perioperative factors. Perioperative factors include procedure duration, perioperative antibiotic prophylaxis, patient preparation and draping techniques, ultraviolet light, operating room (OR) foot traffic, and laminar airflow. The spectrum of pathogens involved in periprosthetic joint infections (PJIs) is broad with the most common being methicillin-resistant staphylococcus aureus (MRSA), coagulase-negative staphylococci, and gram-negative bacilli and enterococcal species.6 Studies have confirmed that operating room (OR) foot traffic is a contributing factor in the occurrence of surgical site infections (SSIs) with $80-90 \%$ of bacterial contaminants in the operating room (OR) coming from the ambient air, specifically by bacterial shedding by operating room (OR) occupants and unsterile equipment.7 Smith et al., found that traffic through operating room (OR) doors increased the expected number of bacteria by $69.3 \%$, and Teter et al., found that large air particles that correlate with bacterial size were elevated significantly on door openings.8.9 In rooms with laminar airflow, the opening and closing of the operating room (OR) door disrupts the airflow in close proximity to the patient. This disruption reportedly allows microbes to enter the airspace above the surgical site, possibly contributing to an increased risk of infection.

Previous studies have been performed looking at the impact of disruptions in laminar airflow as well as foot traffic patterns in surgical procedures. Most studies, when evaluating foot traffic patterns have observed a variety of surgeries (orthopedic, pediatric, general surgery, cardiothoracic, etc.), few have specifically looked at foot traffic patterns for total hip and total knee arthroplasties. One study, performed by Panahi et al., in 2010, evaluated foot traffic patterns during total joint arthroplasties (TJAs) for 80 primary and 36 revision total joint arthroplasties (TJAs) at a high-volume academic center. The authors documented the incidence of door openings as well as the etiology of door openings. They found the average number of door openings for primary cases from incision to closure to be 60 and the average case length was 92 minutes. 5 The mean rate of door openings was 0.65 openings per minute for all cases.5 The largest contributors of door openings were the circulating nurse at $26 \%$ followed by the equipment representative at $20 \%$.

Our study had 3 main objectives: 1) Determine the incidence of door openings during primary total hip arthroplasty (THA) and total knee arthroplasty (TKA) at a non-academic facility, 2) Determine the impact of procedure duration on door openings, and 3) Identify the timing and etiology of door openings in order to develop strategies to reduce the incidence of traffic in the operating room (OR).

\section{Methods}

An observational study was conducted to evaluate operating room (OR) foot traffic patterns (as determined by door openings) for hip and knee arthroplasties. A convenience sample of 16 surgeries from a level 3 non-academic, nonprofit trauma center in the south-west, from June 2019 to July 2019, was collected. To ensure consistency with data collection, an operating room (OR) Assistant, employed by the facility, served as the single observer and data collector. The observer hand-recorded the data throughout each procedure and then transferred it onto a Microsoft Excel spreadsheet. The observer did not contribute to any of the door openings. Operating room personnel were aware of the observer. Approval for this study was obtained from the Perioperative Director of the healthcare system under study.

Cases selected for inclusion met the following criteria: 1) single total hip or knee joint arthroplasty, 2) scheduled during weekdays from 0700-1500, and 3) performed in one of the six identical operating rooms (ORs). Exclusion criteria included total hip and knee arthroplasty (TKA/THA) revision surgeries, partial replacements, and bilateral joint replacements. There were 16 procedures that included 10 inpatient total hip replacements (THRs) and 6 inpatient total knee replacements (TKRs) with a total of 6 different surgeons performing the procedures. Each operating room (OR) had a laminar airflow ventilation system located directly over the operating room table. Door openings were observed only inside the operating rooms (ORs). Door openings were counted during three time periods. Time period one started as soon as the first sterile item was opened and ended when the patient entered the room and the door 
closed. Time period two started from door closure to time of incision. Time period three was from incision to closure. This entire time period is the possible contamination period (PCP).

The data collected included: total number of door openings during each procedure, duration of each procedure, number of door openings during each time period, the door used to enter/exit the operating room (OR) suite (sterile core door versus non-sterile main double doors), and personnel entering/exiting the operating room (OR) during each time period.

\section{Results}

The total number of door openings recorded during 16 cases during the possible contamination period (Time Period $1,2,3$ ) was 1174 . The average number of door openings for all cases during the possible contamination period (PCP) was 73.4 , and the average case length was 147 minutes. The mean rate of door openings was 29.7 per hour and .50 per minute for all cases.

\section{Period 1 Observations (Figure 1a, 1d)}

The total number of door openings recorded during time period 1 (first sterile item was open to when the patient entered the room and the door closed) was 437 . The average number of door openings for all cases during time period 1 was 27 . Thirty seven percent of all door openings occurred during period 1 with surgical technicians and nurses accounting for $31 \%$ each.

\section{Period 2 Observations (Figure 1a, 1d)}

The total number of door openings recorded during time period 2 (door closure to time of incision) was 312 . The average number of door openings for all cases during time period 2 was 20. Twenty-seven percent of all door openings occurred during period 2 with doctors (surgeons and anesthesiologist) being the number one contributor at $40 \%$. Surgeons and anesthesiologist entered initially to verify the team was ready and then exited to scrub and then returned to begin the case. Medical supply representatives accounted for $21 \%$ of openings during this period to communicate with the surgeon and discuss strategies for the procedures. Nurses were responsible for $20 \%$ as they continued to retrieve materials and supplies for the surgery. Surgical technicians were responsible for $19 \%$ as they exit to scrub and re-enter for the procedure.

\section{Period 3 Observations (Figure 1b, 1d)}

The total number of door openings recorded during time period 3 (incision to closure) was 425 . The average number of door openings for all cases during time period 3 was 26 . Thirty six percent of all door openings occurred during period 3 with nurses contributing the most traffic at $38 \%$ due to taking breaks, retrieving lactated ringer and or saline (roughly four times per case), and non-essential foot traffic. Non-essential foot traffic consisted of nurses opening the door to determine if any operating room (OR) staff needed breaks. The medical representative accounted for $36 \%$ as they entered and exited to retrieve implant components. Scrub technicians were responsible for $12 \%$ due to assigned breaks and or shift change. The surgeon contributed $8 \%$ for exiting the operating room (OR) while the nurse closed the incision. X-ray was responsible for $6 \%$ as they would exit the room with equipment. 

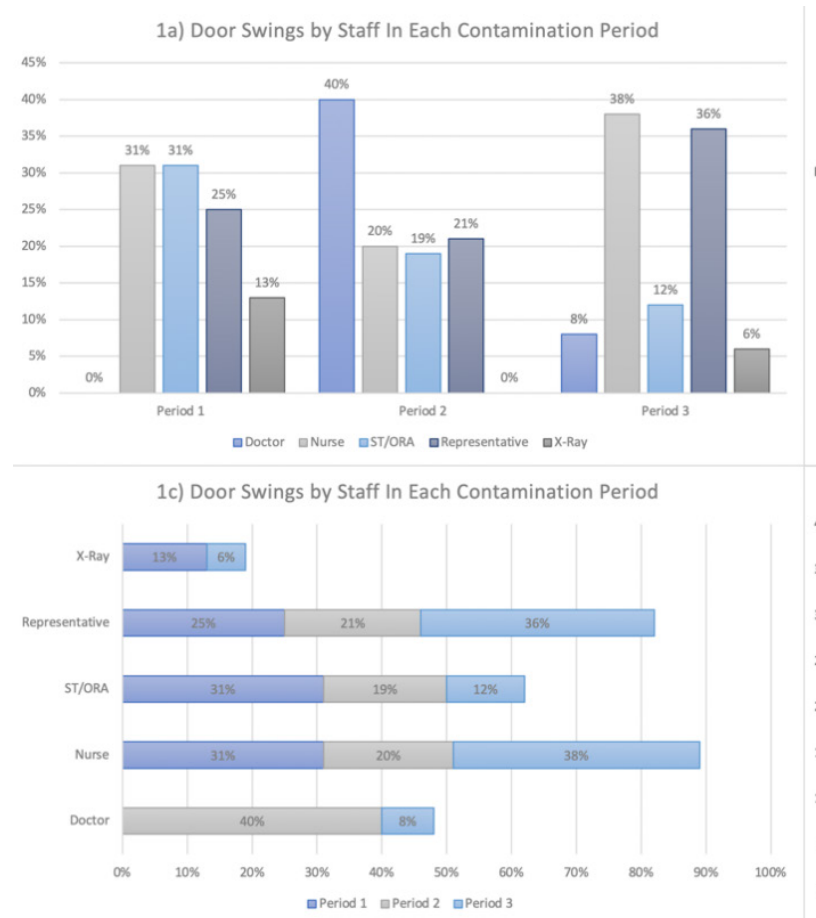

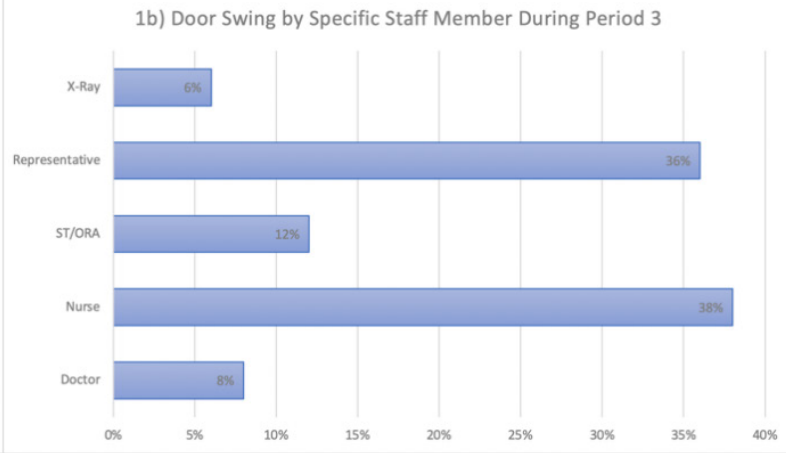

1d) Door Swings During Possible Contamination Period

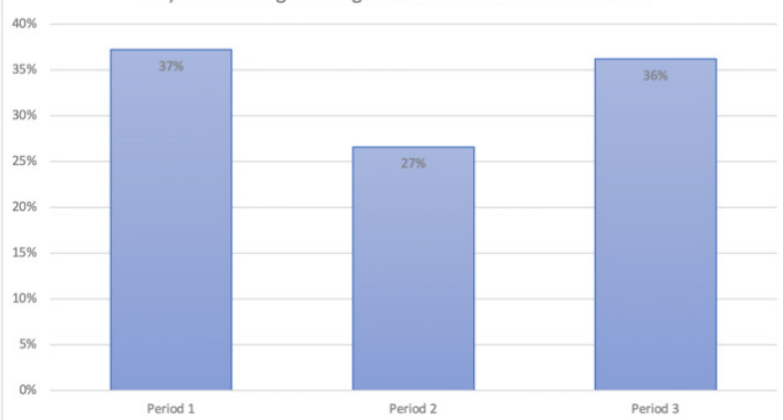

Figure 1. Door Swings in Possible Contamination Period broken out by period and staff. 
Table 1. Case Detail Table. This table provides the details of each case observed by the six different surgeons. Each case includes the total number of swings and duration from the start of the possible contamination period until the end. Included is the average duration and door swings for each surgeon. The possible contamination periods for each surgeon were broken down into door swings to show which period had the most traffic.

\begin{tabular}{|c|c|c|c|c|c|c|}
\hline Case & Swings & $\begin{array}{l}\text { Duration } \\
\text { (hours) }\end{array}$ & $\begin{array}{c}\text { Average } \\
\text { Door Swings }\end{array}$ & $\begin{array}{l}\text { Average Du- } \\
\text { ration }\end{array}$ & \multicolumn{2}{|c|}{$\begin{array}{c}\text { Possible Contamination Period } \\
\text { Per Surgeon }\end{array}$} \\
\hline \multicolumn{7}{|c|}{ Surgeon A } \\
\hline Case 1 & 62 & 2 & \multirow[t]{6}{*}{63} & \multirow[t]{6}{*}{2} & \multirow[t]{2}{*}{ Period 1} & \multirow[t]{2}{*}{134} \\
\hline Case 2 & 62 & 2 & & & & \\
\hline Case 3 & 80 & 2 & & & \multirow[t]{2}{*}{ Period 2} & \multirow[t]{2}{*}{100} \\
\hline Case 4 & 66 & 2.5 & & & & \\
\hline Case 5 & 56 & 2 & & & \multirow[t]{2}{*}{ Period 3} & \multirow[t]{2}{*}{145} \\
\hline Case 6 & 53 & 2 & & & & \\
\hline \multicolumn{7}{|c|}{ Surgeon B } \\
\hline Case 1 & 94 & 2.5 & \multirow[t]{3}{*}{88} & \multirow[t]{3}{*}{3} & Period 1 & 95 \\
\hline Case 2 & 77 & 4 & & & Period 2 & 78 \\
\hline Case 3 & 92 & 3 & & & Period 3 & 90 \\
\hline \multicolumn{7}{|c|}{ Surgeon $\mathrm{C}$} \\
\hline Case 1 & 87 & 3 & \multirow{3}{*}{87} & \multirow[t]{3}{*}{3} & Period 1 & 32 \\
\hline & & & & & Period 2 & 34 \\
\hline & & & & & Period 3 & 21 \\
\hline \multicolumn{7}{|c|}{ Surgeon D } \\
\hline Case 1 & 95 & 3 & \multirow[t]{3}{*}{95} & \multirow[t]{3}{*}{3} & Period 1 & 64 \\
\hline & & & & & Period 2 & 2 \\
\hline & & & & & Period 3 & 29 \\
\hline \multicolumn{7}{|c|}{ Surgeon E } \\
\hline Case 1 & 72 & 1.75 & \multirow[t]{3}{*}{58} & \multirow[t]{3}{*}{1.75} & Period 1 & 57 \\
\hline Case 2 & 50 & 1.5 & & & Period 2 & 61 \\
\hline Case 3 & 52 & 2 & & & Period 3 & 56 \\
\hline \multicolumn{7}{|c|}{ Surgeon F } \\
\hline Case 1 & 81 & 3 & \multirow[t]{3}{*}{88} & \multirow[t]{3}{*}{3} & Period 1 & 55 \\
\hline Case 2 & 95 & 3 & & & Period 2 & 37 \\
\hline & & & & & Period 3 & 84 \\
\hline Case & Swings & $\begin{array}{l}\text { Duration } \\
\text { (hours) }\end{array}$ & $\begin{array}{c}\text { Average } \\
\text { Door Swings } \\
\text { (hours) }\end{array}$ & $\begin{array}{l}\text { Average du- } \\
\text { ration }\end{array}$ & \multicolumn{2}{|c|}{$\begin{array}{c}\text { Possible Contamination Period } \\
\text { Per Surgeon }\end{array}$} \\
\hline
\end{tabular}




\section{Door Openings and Procedure Duration}

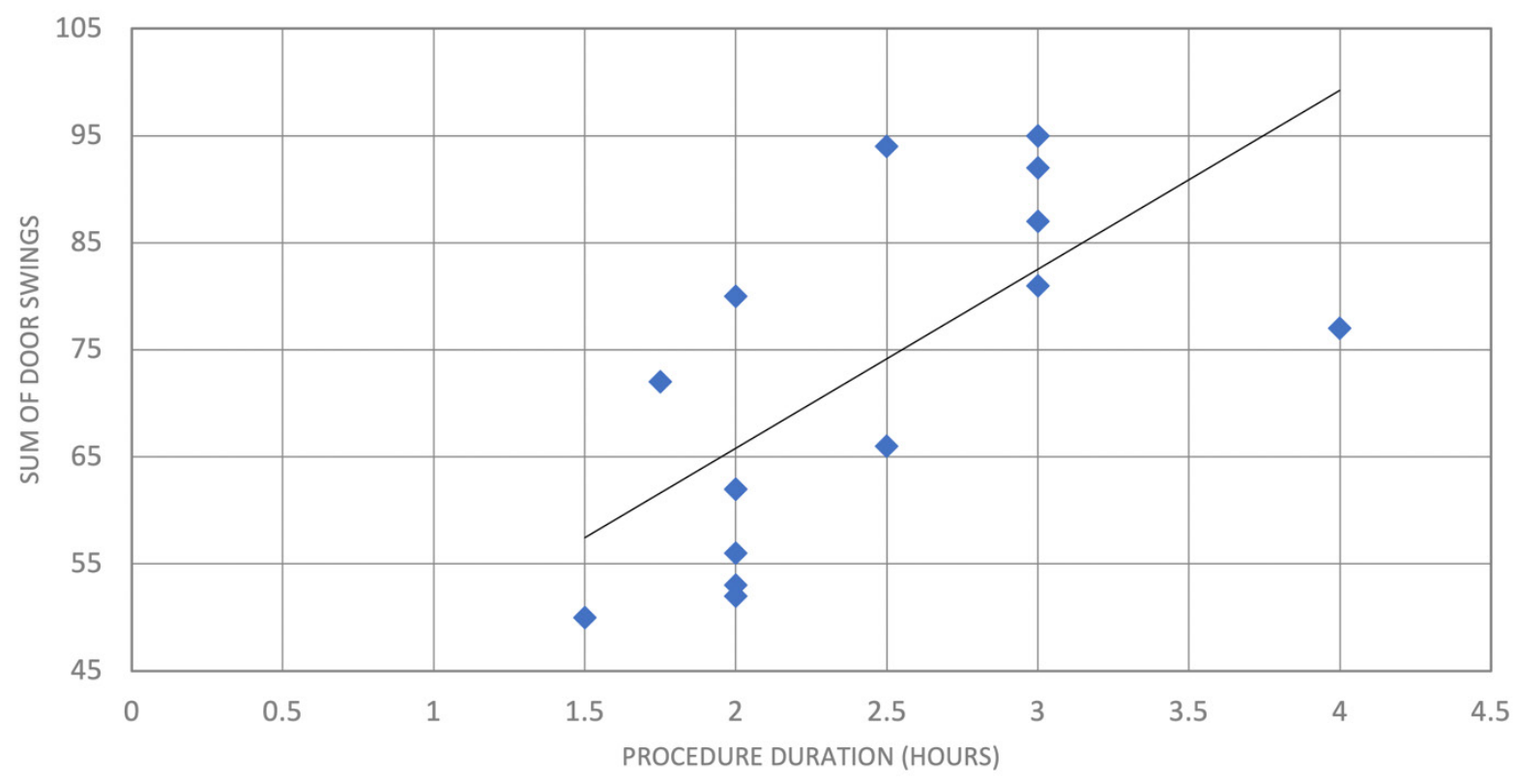

Figure 2. Procedure Duration. Procedure duration has an obvious impact on operating room (OR) foot traffic and door openings. This study found an average of 61 door openings for the possible contamination period (PCP) less than 2 hours, an average of 66 door openings for the possible contamination period (PCP) less than 3 hours and an average of 88 door openings for the possible contamination period (PCP) up to four hours. Door openings increased $25 \%$ per hour for procedures greater than or equal to 3 hours.

\section{Core Door versus Main Door Usage}

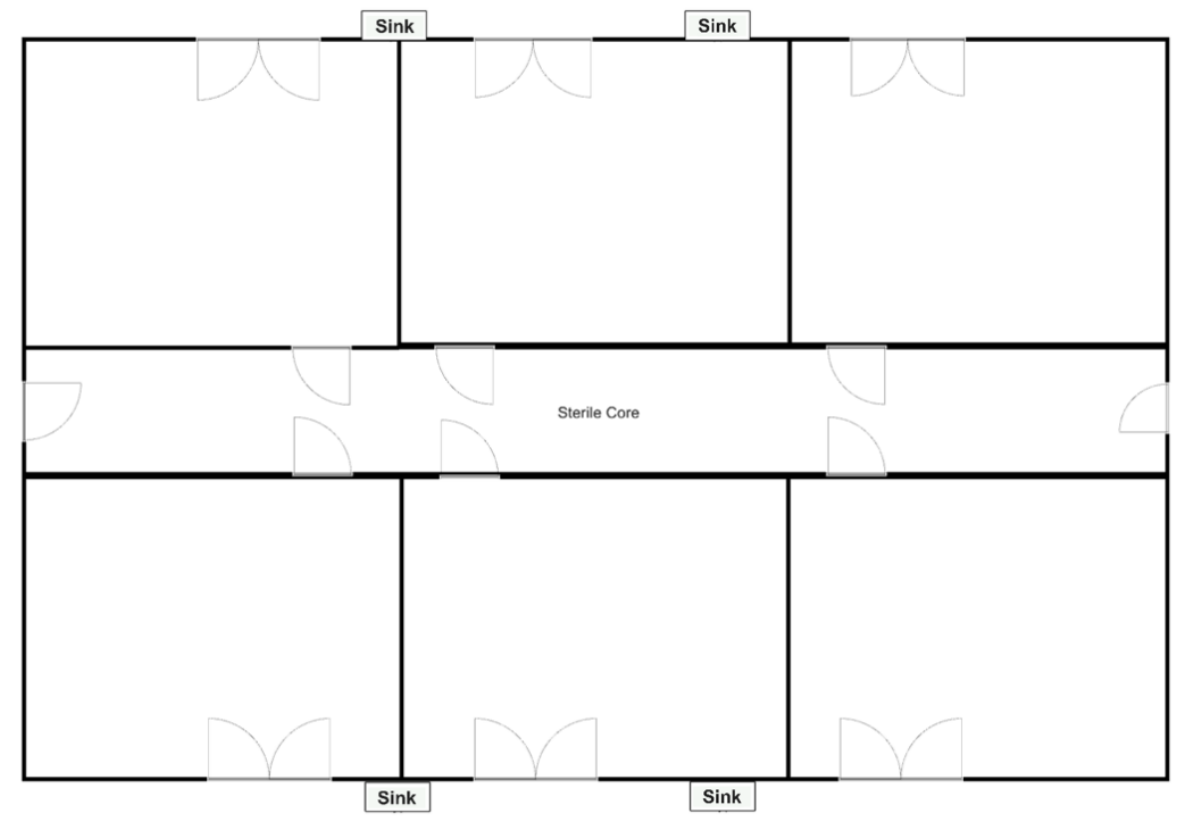

Figure 3. The operating room (OR) Layout. The 6 operating room (OR) suites were identical in layout, with double doors (main door) on the side of the non-sterile hall, allowing the patient and the bed to enter. The core door is the 
passageway from the operating room (OR) into the sterile core. The case carts and sterile supplies, including the implants from the medical sales representative, were located inside of the sterile core. There are four sinks located outside of the operating room suites in the non-sterile hallway.

Core Door Versus Main Door Usage

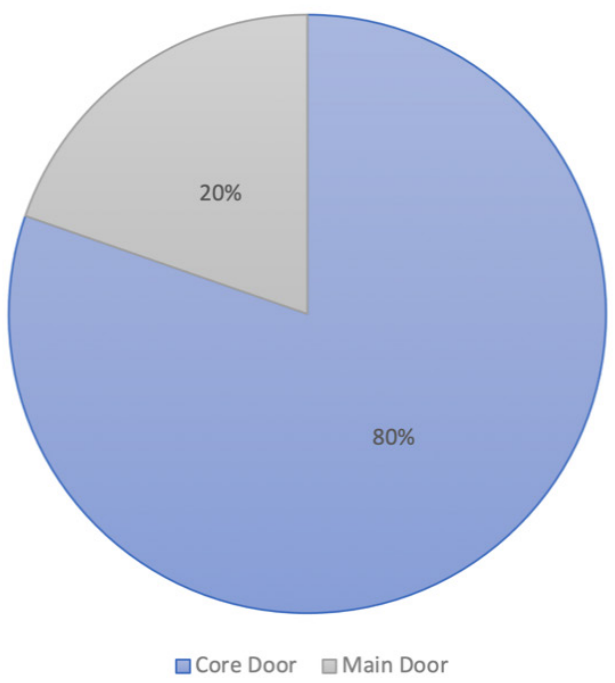

Figure 4. The core door versus main door usage. The hospital highly encourages personnel to enter and exit through the core door during the possible contamination period (PCP). The main door has to be used in two cases: the surgeon and surgical technician scrubbing in, and the patient entering on the gurney. Eighty percent of traffic utilized the core door and $20 \%$ utilized the main doors.

\section{Discussion}

The opening of an operating room (OR) door disrupts the laminar airflow and can increase the bacterial count in the operating room (OR).8,9,10,11 The main objectives of this study were to 1) determine the incidence of door openings during primary total hip and knee arthroplasties (THA and TKAs) at a non-academic facility, 2) determine the impact of procedure duration on foot traffic, and 3) identify the timing and etiology of door openings in order to develop strategies to reduce the incidence of traffic in the operating room (OR).

Overall, for our non-academic facility, the average number of door openings for all primary cases during the possible contamination period was 73.4, and the average case length was 147 minutes. The mean rate of door openings was .50 per minute for all cases. Panahi et al., found an average number of door openings for all primary cases in an academic facility to be 83.2 , and the average case length was 119.5 minutes with the mean rate of door openings at .69 per minute for all cases.

Door openings differed across the surgical periods (time period 1,2, and 3) with the majority of door openings occurring during period $1(37 \%)$ and period 3 (36\%). These results differ from those of Mousavi et al., who found the incision to closure phase (time period 3) to have the fewest door openings.7 This difference is likely due to the fact that the Mousavi study had only 3 orthopedic surgeries out of 28 surgeries they observed. Panahi, et al in their study of 80 primary total joint arthroplasties (TJAs) at an academic facility found that $61 \%$ of door openings occurred after the incision was made.

Results from period one indicated that the majority of door openings can be mitigated by ensuring all resources are located in the operating room (OR) prior to the opening of sterile items. Resources include surgical packs, instrument trays, gowns, gloves, and sutures. Staff should refrain from opening sterile items until all resources are available in the room and the surgeon and patient are ready for surgery, as close to the time of incision as possible. 
The etiology was due to personnel leaving the operating room (OR) to retrieve necessary supplies for the surgery. Medical supply representatives accounted for $25 \%$ of period one's door openings due to verifying the opening of vendor trays and setting up equipment and X-ray technicians accounted for $13 \%$ of traffic due to bringing in the Carm for total hip arthroplasties (THAs). Results from period two indicated that door opening patterns were well controlled with non-essential traffic minimized. The door openings in this period was mainly due to the doctors (surgeon and anesthesiologist) verifying the readiness of the patient and staff, which for this facility, was a non-modifiable factor.

Results from period three indicated that the majority of traffic can be reduced by modifying the door opening patterns of nurses and medical supply representatives. By placing an implant cart in the operating room (OR), door openings could be reduced by $27 \%$. Placing an IV fluid warmer in the operating room (OR) could reduce door openings by nurses by $15 \%$. Determining the need for breaks could be accomplished by using a telephone, rather than opening the core operating room (OR) door, further reducing nursing and surgical technician traffic.

Possible contamination period duration ranged from 1.5 to 4 hours. Door openings increased by $25 \%$ per hour for procedures greater than or equal to 3 hours. The surgeons who averaged two hours had less door swings per hour compared to those who averaged more than two hours. Educating the staff and surgeons on door opening patterns for all periods and how openings increase bacterial count in the OR could be an effective strategy for reducing overall traffic. Developing and implementing an annual surgical site infection prevention module that includes an online competency quiz, would allow facilities to disseminate the information and monitor comprehension of the material. Facilities could install door counters in each room to bring awareness to the number of door swings during procedures and report totals to staff and surgeons monthly.

\section{Conclusion}

Managing door openings in the operating room (OR) is a modifiable risk factor associated with periprosthetic joint infections (PJIs). Understanding door opening patterns during total joint arthroplasties (TJAs) will allow for the implementation of effective strategies to reduce operating room (OR) foot traffic in hospitals and surgery centers. Our results are consistent with those of Panahi, et al, showing clearly that nursing and medical supply representatives contribute to the greatest percentage of door openings during total joint arthroplasties (TJAs) procedures.5 Educating personnel on the correlation between high rates of foot traffic and surgical site infections, properly stocking the operating room (OR) prior to the opening of sterile items (including locating a fluid warmer and implant cart in the OR), reducing overall procedure duration, and limiting door openings to essential openings only, would greatly reduce overall foot traffic. Recommendations for future studies would include identifying differences in door opening behavior for total hip and knee arthroplasty (THA and TKA) procedures and determining what percentage of door openings are related to staff breaks and shift changes.

\section{Limitations}

Limitations to this study included the small number of cases observed and personnel in the operating room (OR) were aware of the observer/data collector. Awareness of the observer can lead to the Hawthorne Effect, resulting in behavior modification from being observed. An additional limitation was that duration of door openings were not timed and the duration of openings varied for every door swing based on the reason for entering/exiting. Another limitation is that we were not able to determine the number of door openings per minute for each surgery period due to not documenting the start and end times for each period. 


\section{References}

1. Kurtz, SM, Lau, E, Schmier, J, Ong, KL, Zhao, K, Parvizi, J. Infection burden for hip and knee arthroplasty in the United States. J Arthroplasty. 2008 Oct;23(7):984-91. https://pubmed.ncbi.nlm.nih.gov/18534466/. Accessed September 1,2020.

2. Wolford, H, Hatfield, K, Paul, P, Yi, S, Slayton, R. The projected burden of complex surgical site infections following hip and knee arthroplasty among adults in the United States, 2020 through 2030. Infect Control Hosp Epidemiol. 2018 October: 39(10): 1189-1195. https://www.ncbi.nlm.nih.gov/pmc/articles/PMC6520991/. Accessed September 1, 2020.

3. Phillips JE, Crane TP, Noy M, Elliott TS, Grimer RJ. The incidence of deep prosthetic infections in a specialist orthopaedic hospital: A 15-year prospective survey. J Bone Joint Surg Br. 2006;88:943 https://pubmed.ncbi.nlm.nih.gov/16799001/. Accessed September 18, 2020.

4. Pulido L, Ghanem E, Joshi A, Purtill JJ, Parvizi J. Periprosthetic joint infection: The incidence, timing, and predisposing factors. Clin Orthop Relat Res. 2008;466:1710 https://pubmed.ncbi.nlm.nih.gov/16799001/. Accessed September 1, 2020.

5. Panahi, P, Stroh, M, Casper, DS, Parvizi, J, Austin, M. Operating room traffic is a major concern during total joint arthroplasty. Clin Orthop Relat Res. 2012 470:2690-2694. https://pubmed.ncbi.nlm.nih.gov/22302655/. Accessed September 18, 2020.

6. Peel, TN, Cheng, AC, Busing, KL, Choong, PFM. Microbiological aetiology, epidemiology, and clinical profile of prosthetic joint infections: Are current antibiotic prophylaxis guidelines effective? Antimicrobial Agents and Chemotherapy. 2012 February 2386-2391. doi:10.1128/AAC.06246-11. https://aac.asm.org/content/56/5/2386 Accessed February 23, 2021.

7. Mousavi, ES, Jafarifiroozabadi, R, Bayramzadeh, S, Joseph A, San D. An observational study of door motion in operating rooms. Building and Environment. 2018 502-507. https://doi.org/10.1016/j.buildenv.2018.08.052. Accessed September 16, 2020.

8. Smith, EB, Raphael, IJ, Maltenfort, MG, Honsawek, S, Dolan, K, Younkins, EA. The effect of laminar air flow and door openings on operating room contamination. The Journal of Arthroplasty 28 (2013) 1482-1485.

https://pubmed.ncbi.nlm.nih.gov/23890828/. Accessed September 16, 2020.

9. Teter, J, Guajardo, I, Al-Rammah, T, Rosson, G, Perl TM, Manahan, M. Assessment of operating room airflow using air particle counts and direct observation of door openings. American Journal of Infection Control 45 (2017) 477-82. https://doi.org/10.1016/j.ajic.2016.12.018. Accessed September 16, 2020.

10. Villafruela, JM, San Jose, JF, Castro F, Zarzuelo, A. Airflow patterns through a sliding door during opening and foot traffic in operating rooms. Build. Environ 109 (2016) 190-198. https://doi.org/10.1016/j.buildenv.2016.09.025. Accessed September 16, 2020.

11. Mousavi, ES, Grosskopf, KR. Airflow patterns due to door motion and pressurization in hospital isolation rooms. Sci. Technol. Built Environ 22 (2016) 379-384. https://doi.org/10.1080/23744731.2016.1155959. Accessed September 16, 2020. 